IN PRACTICE

\title{
Aetiology of sexually transmitted infections and response to syndromic treatment in southwest Uganda
}

\author{
J M Pickering, J A G Whitworth, P Hughes, M Kasse, D Morgan, B Mayanja, L Van der Paal, \\ P Mayaud
}

Sex Transm Infect 2005;81:488-493. doi: 10.1136/sti.2004.013276

See end of article for authors' affiliations

Correspondence to: Julie Pickering, Medical Research Council Clinical Trials Unit, 222 Euston Road, London NW1, UK; jmb@ctu.mrc.ac.uk

Accepted for publication 11 March 2005

Objective: To determine the aetiology of genital ulcers and discharges in rural south western Uganda and to assess response to syndromic treatment.

Method: A longitudinal, prospective study using laboratory testing and questionnaires to evaluate 561 adult men and women presenting with clinically verified genital ulcers, urethral, or vaginal discharge at a general outpatient clinic and two health centres between December 1999 and July 2001.

Results: One third of patients had genital ulcers and two thirds discharges. There was good response to treatment in $461 / 508$ patients (90.7\%). Herpes simplex virus type 2 was found in 95/217 (43.8\%) genital ulcers. In $24.1 \%$ of ulcer cases there was also a genital discharge. HIV seropositivity was high in ulcer cases (63.2\%), with significantly more HSV2 and secondary bacterial infection than in seronegative cases. Neisseria gonorrhoeae was found in 135/204 (66.2\%) male genital discharges. Female genital discharges were mostly associated with bacterial vaginosis (36.1\%), Trichomonas vaginalis (18.9\%), and candidiasis (18.6\%).

Conclusions: The aetiological pattern of STI syndromes reported will help inform revision of national STI guidelines. The importance of herpes simplex virus type 2, the variation in causes of genital ulcers according to HIV serostatus, the high frequency of multiple infections and secondary bacterial contamination of genital ulcers are notable. These results help explain the lack of effect of an STI intervention on HIV incidence in a recent trial in this area.

S exually transmitted infections (STIs) are important causes of mortality and morbidity in African populations resulting in, among other things, adverse birth outcomes, neonatal and infant infections, ectopic pregnancy, anogenital cancer, infertility, pelvic inflammatory disease, and death. ${ }^{1}$ As such, they merit priority for treatment in their own right. In addition, it is generally accepted that genital ulcers and other STIs are associated with an increased risk of HIV transmission. ${ }^{2}$ There is evidence that in some circumstances in sub-Saharan Africa the treatment and management of STIs can be used as a public health strategy to lower the incidence of HIV infection. ${ }^{3}$

Many low income countries with limited laboratory services, especially in rural areas, have adopted syndromic treatment of STIs. With this approach patients are treated on the basis of groups of symptoms and signs (syndromes) rather than by identifying specific STI pathogens. Because several STIs can cause a particular syndrome, all likely pathogens need to be treated at the same time. Treatment protocols therefore rely on an understanding of the local aetiological pattern of STIs. The syndromic approach can be learned and applied by primary healthcare workers and allows the treatment of symptomatic patients in one clinic visit. However, drawbacks to this approach include overtreatment and therefore costly drug wastage and side effects, a reliance on the correct organisms being targeted by the treatment algorithms, correct drugs being available, and inadequate care for asymptomatic STI patients.

Reported prevalence and aetiological agents for STIs vary widely by geographical area and population. For example, the epidemiology of genital ulceration in Africa has changed over time with a drop in chancroid and syphilis and an increase in genital herpes, which may be real or the result in part in improved techniques for diagnosing herpes simplex virus (HSV) by HIV control programmes. ${ }^{4}$
In the few areas where longitudinal data are available, there is evidence of a shift in the aetiology of genital ulcers towards HSV-2. For example, the proportion of genital ulcer disease (GUD) cases that were herpes culture positive increased from $11 \%$ in $1986-8$ to $21 \%$ in $1990-2$ among HIV positive cases in Rwanda. ${ }^{5}$ A study among miners in Carletonville, South Africa, reported that the proportion of ulcers caused by herpes increased from $1.3 \%$ in 1986, to $10 \%$ in 1994 and $36 \%$ in 1998, while conversely the prevalence of chancroid decreased from $68.5 \%$ to around $50 \%$, and the proportion of patients with a serological syphilis diagnosis decreased from $26 \%$ in 1986 to $18 \%$ in 1994 and only $11 \%$ in $1998 .{ }^{67}$ In Durban, the proportion of ulcers caused by HSV-2 increased from $11 \%$ in 1991 , to $36 \%$ in 1994 , to $48 \%$ in $2002 .{ }^{8}$ Similar findings have been observed in Botswana, ${ }^{9}$ Lesotho, ${ }^{10}$ and Kenya. ${ }^{11}$

This study was designed to determine the prevalence of a variety of STI pathogens among patients presenting at rural health clinics in Uganda with complaints of genital ulcers (GU), and urethral and vaginal discharge. We assessed the effectiveness of pre-existing national syndromic treatment protocols against the most prevalent causes of STI in this population and identified risk factors for various reproductive tract infections.

\section{METHODS}

Patients presenting at a rural general outpatient clinic and two rural health centres taking part in the Masaka

Abbreviations: BV, bacterial vaginosis; $\mathrm{CT}$, Chlamydia trachomatis; GU, genital ulcers; FGD, female genital discharge; GUD, genital ulcer disease; HD, Haemophilus ducreyi; HSV, herpes simplex virus; IQR, interquartile range; LCR, ligase chain reaction; MGD, male genital discharge; NG, Neisseria gonorrhoea; PCR, polymerase chain reaction; RPR, rapid plasma reagin; STI, sexually transmitted infections; TP, Treponema pallidum; TV, Trichomonas vaginalis 
Intervention Trial ${ }^{12}$ were registered for evaluation between December 1999 and January 2001. Those complaining of GU or genital discharge were invited to take part and a patient information sheet was read to them by a clinician. We excluded those under 15 years old, pregnant with a genital discharge, or repeat attendance with a new STI within 1 month. After giving informed written consent, which also covered follow up visits at home, patients were interviewed and examined. At interview participants answered questions on personal characteristics, current and past STIs, treatment seeking and sexual behaviour. Participants underwent a full general and genital examination where details were taken of GU and discharge: the location and size (measured with a disposable paper ruler) of the largest ulcer in patients with genital ulceration; the amount and type of urethral discharge in male genital discharge (MGD) cases; and vulval, vaginal, and cervical inflammation, type of vaginal or cervical discharge in female genital discharge (FGD) cases. Clinical and interview findings were recorded onto precoded questionnaires. Privacy and comfort were maintained at all times. A syndromic diagnosis was made of GU, MGD, or FGD.

\section{Sample collection}

In GU cases, serous fluid squeezed from the largest ulcer was sampled with two swabs; one was agitated in polymerase chain reaction (PCR) transport medium and the other into Amies transport medium for bacterial culture. At the general clinic only, blood was taken for HIV and syphilis serology.

In cases of MGD, a urethral swab was taken, applied on gonoline media, and a smear made. A 2 hour post-micturition urine sample was also collected for analysis by ligase chain reaction (LCR) and TV-Inpouch culture.

FGD investigations were only done in the general clinic because of a lack of facilities in the other health centres. Two high vaginal swabs were collected; one was agitated into a TV InPouch and the second smeared onto a glass slide for Gram staining. An endocervical swab for LCR was taken. At the general clinic specimens were delivered to the laboratory immediately, while at the health centres they were stored under appropriate conditions and transported to the laboratory each day. A laboratory form was completed for each patient and specimens were labelled, processed and examined to establish the aetiological agents.

\section{Laboratory testing}

PCR testing was done for HSV type 2, Haemophilus ducreyi (HD) and Treponema pallidum (TP). The multiplex PCR test consisted of DNA probe coated microwells, reagents, and collection swabs developed for research use only (Roche Diagnostic Systems, USA); and a primers/mastermix solution supplied by Dr Ian Maclean (University of Manitoba, Canada). An internal control plate was included to allow a check for inhibition. Bacterial cultures were performed using sheep blood and Mackonkey agar plates incubated at $37^{\circ} \mathrm{C}$ aerobically for 48 hours.

Samples were tested for HIV infection twice by ELISA (Recombigen HIV-1/HIV-2 EIA, Trinity Biotech Ltd, Ireland and Wellcozyme HIV Recombinant EIA, Murex Biotech Ltd, UK). Samples with discordant results were further tested by western blot (Cambridge Biotech, Calypte Biomedical Corporation, Rockville USA). Syphilis testing was performed by rapid plasma reagin (RPR) and TPHA (Biotech RPR/TPHA kits, Biotech Laboratories Ltd, Suffolk, UK).

Neisseria gonorrhoea (NG) and Chlamydia trachomatis (CT) detection was performed by the ligase chain reaction (Abbott Laboratories, IL, USA). Gonoline cultures (Biomerieux, Durham, USA) were incubated for up to 36 hours at $37^{\circ} \mathrm{C}$. Positive cultures were tested for antimicrobial resistance using Muller-Hinton/bovine haemoglobin media supplemented by $20 \mathrm{mg} / \mathrm{ml} \mathrm{NAD}$ using E-test strips. Plates were read after 24 hours of incubation at $37^{\circ} \mathrm{C}$ in a $\mathrm{CO}_{2}$ rich environment. Interpretation of E-test MIC results was based on WHO/CDC reference breakpoints.

Trichomonas vaginalis (TV) was detected by TV-Inpouch cultures (Biomed Diagnostics Inc, San Jose, CA, USA) after centrifugation at $500 \mathrm{rpm}$ for 5 minutes. The Gram stained smear was evaluated for bacterial vaginosis (BV) using Nugent's score, ${ }^{13}$ and the presence of budding and hyphal yeasts was also noted.

\section{Treatment and follow up}

Treatment was provided according to national guidelines for STI management. First line treatment for GU syndrome is $500 \mathrm{mg}$ ciprofloxacin (immediately) and 2.4 MU benzathine penicillin (intramuscular, immediately). First line treatment for MGD syndrome is $500 \mathrm{mg}$ ciprofloxacin (immediately) and $100 \mathrm{mg}$ doxycycline (twice daily for 7 days). First line treatment for FGD syndrome is $500 \mathrm{mg}$ ciprofloxacin (immediately), $100 \mathrm{mg}$ doxycycline (twice daily for 7 days), $2 \mathrm{~g}$ metronidazole (immediately), and $500 \mathrm{mg}$ clotrimazole pessaries. Patients were counselled on compliance to the drugs and avoidance of re-infection. Coupons were issued for partner notification, and for follow up appointment 1 week later and weekly thereafter until the patient was cured. At follow up a questionnaire was administered asking the participants about any current symptoms, to what extent they complied with drug treatments, and a clinical examination was conducted to look for further signs of ulcers and discharge. An outcome assessment was made by the clinician at the 1 week follow up visit of clinically cured, improving, treatment failure or other. A healed genital ulcer, or size reduction of $90 \%$ or more was assessed as cured; a $50-90 \%$ reduction was assessed as improving; while treatment failure was an ulcer that was still $50 \%$ or more in size than it was at enrolment. MGD patients were assessed as cured if they were found with no symptom or discharge on milking of the urethra; slight symptoms of dysuria or discharge but no discharge on milking was assessed as improving; while those with more severe symptoms and discharge on milking were considered to have a treatment failure. FGD cases were considered cured if no symptoms or discharge was seen; a reduction in symptoms and discharge was considered to be improving; and an assessment of treatment failure was if the discharge had not improved. In cases of treatment failure second line treatment was given after 7 days.

Participants not returning for follow up were visited at home. A field worker administered a questionnaire, but did not perform a clinical examination. The field worker recorded the patient's self assessment of cure, whether they had fully complied with the medication, any current symptoms, and reasons for not returning for their follow up visit. Home visits were initiated for participants enrolling after September 2000 to improve information on response to treatment.

\section{Statistical analysis}

Questionnaires were double data entered onto a FoxPro database and statistical analyses in Stata 6 (Stata Corporation, TX, USA) were conducted after data cleaning. Descriptive statistics were performed for all participants at enrolment, comparing those in each syndrome group, with significance tests where appropriate.

\section{RESULTS}

There were 561 participants with a median age of 28 (IQR 2336). Of 261 males, $75(28.7 \%)$ presented with GU, 175 (67.0\%) with MGD, and 11 (4.2\%) with both. Of 300 women, $86(28.7 \%)$ presented with GU, $174(58.0 \%)$ with FGD, and 40 $(13.3 \%)$ with both. Of the 561 individuals, $372(66.3 \%)$ 
Table 1 Aetiology of genital ulcers and treatment outcome

\begin{tabular}{|c|c|c|c|c|c|c|c|c|c|}
\hline & \multirow[b]{2}{*}{ Total (\%) } & \multicolumn{3}{|c|}{ HIV statust } & \multicolumn{4}{|c|}{ Treatment outcome (first line) } & \multirow[b]{2}{*}{ Non-returner } \\
\hline & & Positive & Negative & p Value & Cured & Improving & $\begin{array}{l}\text { Treatment } \\
\text { failure }\end{array}$ & Other & \\
\hline TP alone & $7(3.5)$ & 1 & 3 & & 2 & 1 & 0 & 0 & 4 \\
\hline HD alone & $5(2.5)$ & 1 & 0 & & 0 & 3 & 0 & 0 & 2 \\
\hline HSV alone & $80(39.6)$ & 41 & 17 & & 29 & 15 & 12 & 1 & 23 \\
\hline HSV and $S$ aureus & $12(5.9)$ & 6 & 0 & & 5 & 5 & 0 & 0 & 2 \\
\hline$S$ aureus alone & $21(10.4)$ & 6 & 7 & & 7 & 6 & 0 & 1 & 7 \\
\hline Strep pyogenes alone & $2(1.0)$ & 1 & 1 & & 1 & 0 & 0 & 0 & 1 \\
\hline $\mathrm{HSV}$ and HD & 110.5 & 0 & 0 & & 0 & 0 & 0 & 0 & 1 \\
\hline HSV and Strep pyogenes & $2(1.0)$ & 1 & 0 & & 0 & 2 & 0 & 0 & 0 \\
\hline No organism identified & $72(35.6)$ & 30 & 21 & & 40 & 6 & 2 & 0 & 24 \\
\hline Total & 202 & 87 & 49 & 0.309 & 84 & 38 & 14 & 2 & 64 \\
\hline
\end{tabular}

returned for follow up. Seventy six $(40.2 \%)$ of the nonreturners were enrolled before September 2000 so were not visited at home. Of the remaining 113, 65 (57.5\%) were located, visited, and interviewed.

\section{Genital ulcers}

Full laboratory results were available for 202 (95.3\%) of 212 cases (table 1). In some individuals more than one organism was identified, leading to 217 diagnoses being associated with a treatment outcome. Fifteen (7.4\%) individuals had more than one pathogen identified on testing. Out of 94 cases negative on multiplex PCR, pure growths of $S$ aureus were seen in $21(22.3 \%)$ cases and Strep pyogenes in two $(2.1 \%)$ cases. The aetiological patterns were similar in males and females.

Of the $138(68.3 \%)$ patients returning for follow up, 122 $(88.4 \%)$ were assessed as clinically cured or improving after first line treatment. Eighteen of the 21 (85.7\%) non-returners who were followed up said they were cured or partially cured. Overall, 140/159 (88.1\%) participants with follow up information were cured or improving after first line treatment.

In all, 145 participants had blood samples taken for HIV testing. There was one indeterminate result after western blot. Of the 144 tests with a definite serostatus, 91 (63.2\%) were HIV positive. HIV positive participants were older than HIV negative participants, median age (IQR) 30 (25-39) versus 26 (20-36) respectively (Wilcoxon rank sum $\mathrm{p}=0.03$ ). Full laboratory information was available for 136 (94.4\%) of these individuals. TP was detected in four $(2.9 \%)$ cases (one HIV positive and three HIV negative participants), HD in one HIV positive case, and HSV2 in 65 cases ( 48 HIV positive and 17 HIV negative). HSV2 was more common in HIV positive participants than HIV negative participants (Fisher's exact two sided $p=0.031$ ). All secondary bacterial contaminations were identified in HIV positive participants (six $S$ aureus and one Strep pyogenes). After first line treatment $94.6 \%$ of HIV negative participants reported to be cured or improving compared with $80.6 \%$ of HIV positive participants (Fisher's exact two sided $\mathrm{p}=0.078$ ).

The same 145 participants had blood samples taken for syphilis serology; 31 (21.4\%) were TPHA positive only, 13 $(9.0 \%)$ were RPR positive only, and eight $(5.5 \%)$ were dually TPHA and RPR positive.

\section{Male genital discharge}

Full laboratory results were available for 183 (98.4\%) out of 186 men. In some individuals more than one organism was identified leading to 204 diagnoses being associated with a treatment outcome (table 2 ). Twenty (10.9\%) individuals had more than one pathogen identified on testing. All NG isolates were sensitive to first line treatment with ciprofloxacin.

Of the $100(54.3 \%)$ participants returning for follow up, 96 $(96.0 \%)$ were cured or improving after 1 week of follow up. Thirty two $(91.4 \%)$ of the 35 non-returners who were followed up said they were cured or partially cured. Overall, $128 / 135(94.8 \%)$ participants with follow up information were cured or improving after first line treatment.

\section{Female genital discharge}

Full results of laboratory investigations were available for 206 $(96.3 \%)$ out of 214 women. In some individuals more than one organism was identified leading to 281 diagnoses associated with a treatment outcome (table 3). Sixty two $(30.1 \%)$ individuals had more than one pathogen identified on testing.

World Health Organization suggested risk factors for cervical infections ${ }^{14}$ were assessed in the 18 women $(8.7 \%)$ who had either NG or CT. Only age $<21$ years was

Table 2 Aetiology of male genital discharges and treatment outcome

\begin{tabular}{|c|c|c|c|c|c|c|}
\hline & \multicolumn{6}{|c|}{ Treatment outcome (first line) } \\
\hline & Cured & Improving & Treatment failure & Other* & Non-refurner & Total (\%) \\
\hline TV alone & 1 & 1 & 0 & 1 & 4 & $7(3.8)$ \\
\hline NG alone & 51 & 7 & 0 & 1 & 56 & $115(62.8)$ \\
\hline CT alone & 2 & 0 & 1 & 0 & 1 & $4(2.2)$ \\
\hline NG and TV & 2 & 0 & 0 & 1 & 3 & $6(3.3)$ \\
\hline NG and $C T$ & 7 & 0 & 0 & 0 & 6 & $13(7.1)$ \\
\hline TV and NG and CT & 0 & 0 & 0 & 0 & 1 & $1(0.5)$ \\
\hline No organism identified & 18 & 7 & 0 & 0 & 12 & $37(20.2)$ \\
\hline Total & 81 & 15 & 1 & 3 & 83 & 183 \\
\hline
\end{tabular}

TV, Trichomonas vaginalis; NG, Neisseria gonorrhoeae; CT, Chlamydia trachomatis.

*Two were re-infections and one was non-compliant with treatment. 
Table 3 Aetiology of female genital discharges and treatment outcome

\begin{tabular}{|c|c|c|c|c|c|c|}
\hline & \multicolumn{6}{|c|}{ Treatment outcome } \\
\hline & Cured & Improving & Treatment failure & Other & Non-returner & Total \\
\hline TV alone & 3 & 6 & 0 & 0 & 4 & 13 \\
\hline BV alone & 17 & 16 & 0 & 1 & 9 & 43 \\
\hline Candida alone & 16 & 4 & 1 & 0 & 4 & 25 \\
\hline NG alone & 1 & 2 & 1 & 0 & 1 & 5 \\
\hline TV and BV & 12 & 5 & $i$ & 0 & 8 & 26 \\
\hline TV and candida & 1 & 0 & 1 & 0 & 0 & 2 \\
\hline TV and NG & 0 & 0 & 0 & 0 & 1 & 1 \\
\hline $\mathrm{BV}$ and candida & 6 & 4 & 4 & 0 & 5 & 19 \\
\hline Candida and NG & 1 & 0 & 0 & 0 & 0 & 1 \\
\hline $\mathrm{BV}$ and $\mathrm{TV}$ and candida & 1 & 0 & 0 & 0 & 2 & 3 \\
\hline$B V$ and $T V$ and $N G$ & 1 & 2 & 0 & 1 & 3 & 7 \\
\hline $\mathrm{BV}$ and $\mathrm{TV}$ and $\mathrm{CT}$ & 1 & 0 & 0 & 0 & 0 & 1 \\
\hline $\mathrm{BV}$ and candida and NG & 1 & 0 & 0 & 0 & 1 & 2 \\
\hline No organism identified & 24 & 18 & 1 & 0 & 15 & 58 \\
\hline Total & 85 & 57 & 9 & 2 & 53 & 206 \\
\hline
\end{tabular}

significantly associated with these infections (13/183 aged $>=21$ compared with 5/25 aged $<21$, Fisher's exact $p=0.048$ ). Being single (Fisher's exact $p=0.536$ ), having had $>1$ partner in the last 3 months (Fisher's exact $\mathrm{p}=1.000$ ) and having had a new partner in the last 3 months (Fisher's exact $\mathrm{p}=0.252$ ) were not associated with an increased prevalence of cervical infections.

A diagnosis of BV was associated with TV infection (37\% with BV also had TV compared with $16 \%$ of those without $\mathrm{BV}$; Fisher's exact $\mathrm{p}<0.001)$. BV and candida were not found to be associated: $23 \%$ with BV also had candidiasis compared with $17 \%$ of those without BV (Fisher's exact $\mathrm{p}=0.5$ ).

Of the $158(73.8 \%)$ participants returning for follow up, $147(93.0 \%)$ were cured or improving after first line treatment. Thirteen $(76.5 \%)$ of the 17 non-returners who were followed up said they were cured or partially cured. Overall, 160/175 (91.4\%) participants with follow up information were cured or improving after first line treatment.

\section{Genital ulcer and genital discharge}

Full results of laboratory investigations were available for 45/ $51(88.2 \%)$ participants. Thirty two $(80 \%)$ of 40 females with full results were HIV positive compared with only one in five $(20 \%)$ males with full laboratory results (Fisher's exact $\mathrm{p}=0.014)$. TP was identified in one $(2.2 \%)$ case, HD in two $(4.4 \%)$ cases, HSV in $22(48.9 \%)$ cases, NG in seven $(15.6 \%)$ cases, TV in $11(24.4 \%)$ cases, $S$ aureus in four $(8.9 \%)$ cases, Strep pyogenes in one $(2.2 \%)$ case, BV in $18(40.0 \%)$ cases, candida in $12(26.7 \%)$ cases, and no organism was identified in three $(6.7 \%)$ cases. One organism was identified in 11 $(24.4 \%)$ cases, two in $21(46.7 \%)$ cases, three were identified in eight $(17.8 \%)$ cases, and four in two $(4.4 \%)$ cases. Twenty seven out of 31 participants who returned for follow up after l week were observed to be either cured or improving. Six of eight non-returners who were followed up were also reported to be cured or improving. Altogether, 33/39 (84.6\%) participants with follow up information were cured or improving after first line treatment.

\section{DISCUSSION}

In this study we found about one third of patients presented with GU and two thirds with genital discharges. The distributions were similar between the sexes. This compares with two population based studies in southwest Uganda which reported slightly higher rates of GU than discharges. ${ }^{12}{ }^{15}$ It is noteworthy that one quarter of cases of GU also had a discharge. This degree of overlap of syndromes was surprisingly high and is increasingly being recognised in studies in sub-Saharan Africa. ${ }^{16}$ We recommend that when both syndromes co-exist the appropriate treatment be clearly outlined to health workers (for example, for males give single doses of ciprofloxacin and benzathine penicillin and a 7 day course of doxycycline). The findings from this study have some practical implications, in particular in the preparation of national STI guidelines, which often do not take into account information from rural areas, and in guiding the choice of antibiotics that can target more than one pathogen.

HSV2 was the most common cause of GU in this population (44\% of diagnoses). This is in close agreement with previous studies in urban and rural Uganda. ${ }^{18}$ Genital herpes has been recognised as an increasingly important cause of GU throughout sub-Saharan Africa. ${ }^{4}$ In contrast, only a minority of cases could be attributed to the "classic" causes of ulcer in Africa: syphilis and chancroid accounting for only $6 \%$ of GU.

Several explanations have been proposed for this apparent increase in HSV2 as a cause of GU in Africa. Firstly, these shifts in GU aetiology could in part reflect changes in detection rates based earlier on clinical definitions or culture identification to the more common use of molecular diagnostic methods in recent studies-although this explanation should equally apply to bacterial causes of GU. Secondly, decreases in the prevalence of bacterial causes of GU may have been the result of successful bacterial STI control measures such as widespread adoption of STI syndromic management and targeted interventions with high risk populations, leading to large decreases in chancroid and syphilis, thereby inducing a relative increase in HSV2 as a cause of GU. Thirdly, modelling studies have suggested that behavioural changes (increased condom use or reduction of partner change) could have made an important contribution to the relative increases in genital herpes, although some of the observed increases have occurred where behaviour changes had not been noted (for example, South Africa). ${ }^{19}$ Finally, and importantly, the emergence of genital herpes could be a consequence of the complex interaction between HIV and HSV2. Immunosuppression during advanced HIV disease can increase the duration, severity, and incidence of herpetic recurrences, leading to an increased herpes ulcer load and greater transmissibility to uninfected populations. ${ }^{20}$ In advancing HIV epidemics, a larger pool of dually infected patients may exist, contributing to the greater transmission of both viruses, accounting for a large proportion of new HIV cases. $^{21}$ For this reason, measures to control HSV2 are urgently needed. ${ }^{20}$

The results of this study highlight the question of whether an antiviral drug for HSV2 should be added to treatment 
algorithms for genital ulcers in high prevalence areas, as recently recommended by the World Health Organization. ${ }^{22}$ However, all antiviral regimens are multidose, are suppressive rather than curative, and have limited effect unless taken in the first one or two days in the course of an episode. In addition, most patients in this study reported recovery without specific treatment. It is thought that there is an important interaction and facilitating role of HSV in HIV acquistion and transmission, ${ }^{20}$ and a recent study has demonstrated that suppressive therapy with valaciclovir can reduce the transmission of HSV2 in discordant couples. ${ }^{23}$

This study also highlights the importance of secondary bacterial contamination of GU. Pure growths of $S$ aureus or Strep pyogenes were isolated from over $12 \%$ of GU when multiplex PCR was negative. Our clinical impression was that these bacterial contaminations mainly occurred in old herpetic ulcers, traumatic abrasions, and excorations. While they are not STIs, they are important because they will delay the healing of ulcers leaving the patient at risk of transmitting HIV infection. The presence of bacterial contamination of GU has been previously overlooked, but is probably common in most areas with a hot and humid climate and limited access to clean water and soap. ${ }^{24}$

HIV seropositivity was recorded in $63 \%$ of participants presenting with GU. This is extremely high when compared with the HIV prevalence of about $8 \%$ in the general population in this area. ${ }^{13}$ This is of particular concern because such patients have the potential to transmit and acquire HIV through their GU, especially if their GU is not adequately treated..$^{25}$

We noted a significantly different pattern of aetiologies of GU according to HIV serostatus. There was relatively more HSV2 and secondary bacterial contamination among seropositive participants. It has previously been reported that herpes ulcers are slow to heal and have more relapses in HIV infection, ${ }^{26}$ which may account for the higher prevalence found in this study, and the immunocompromised nature of the patients may contribute to the higher frequency of secondary bacterial contamination of lesions.

We recorded a good response to treatment of GU at 1 week. Response to treatment tended to be worse in HIV seropositive participants, but was still over $80 \%$. It is reassuring that even streptococcal and staphylococcal infections responded to treatment with a single dose of ciprofloxacin, or resolved spontaneously. Of ulcers associated with HSV2, 81\% (56/59) were reportedly cured or improving after 1 week. Presumably this was spontaneous resolution of the episodes and was unrelated to treatment. There was relatively high drug wastage in GU cases in this study as $40 \%$ of them were only infected with HSV which would not respond to any of the treatments offered. However, the antibiotics given to these participants may have prevented bacterial superinfection, and so accelerated cure, reducing the amount of time with increased risk of HIV transmission.

FGD was associated with BV in over one third of cases, with TV and candidiasis each associated with about $20 \%$ of cases. There was a good response to treatment of FGD and MGD after 1 week. Similar findings have been reported from South Africa ${ }^{27}$ and Tanzania. ${ }^{18}$

NG or CT infections were found in less than 10\% of FGD and, as in male discharges, NG was observed at least seven times more frequently than CT. The prevalence of CT observed in women with FGD in this study was very low $(0.6 \%)$ in the light of a population prevalence in urine of $1.7 \%$ in 13-39 year olds recorded in Masaka District. ${ }^{12}$ Even this figure from the Masaka Intervention Trial is low compared with other reports from the region: $3.8 \%$ in a population based study in the Rakai district of Uganda ${ }^{15} ; 9 \%$ in a clinic population in Nairobi, Kenya ${ }^{28}$; and $3.2 \%$ in a study of women
Key messages

- Information on local aetiological patterns of STI syndromes and responses to treatment are required to inform the development and regular revision of appropriate national STI syndromic treatment guidelines

- In Uganda, herpes simplex type 2 was associated with more than $40 \%$ of genital ulcers

- Secondary bacterial contamination of ulcers with Staph aureus and Strep pyogenes was common, especially in those with HIV infection

- Multiple infections and a combination of genital ulcers and discharges were unexpectedly common

recruited from agricultural plantations in western Kenya. ${ }^{29}$ The prevalence of NG was 7\% in women with FGD compared with $0.9 \%$ in $13-39$ year olds in Masaka District. ${ }^{12}$ This is within range of other studies: $1.6 \%$ in a population based study in the Rakai district of Uganda ${ }^{15}$ to $7 \%$ in a Nairobi clinic population. ${ }^{28}$ It appears that NG is associated with vaginal discharge in this population while CT is not. This may be because NG is more symptomatic so women are more likely to present to a clinic with it. Other possible reasons include the longer duration of CT infection compared to NG, meaning that the incidence of CT might be low relative to the population prevalence; or previous treatment with cyclines, which are widely available in Uganda. We have no reason to doubt the LCR assays used on specimens taken during the study: the assays were on specimens taken from endocervical swabs, which is the most sensitive method of detection. If these findings on CT are replicated in other settings in Uganda, the authorities might want to consider omitting doxycycline from first line treatment for vaginal discharge, but this would have to be carefully monitored.

In this paper we have described the aetiological pattern of genital ulcers and discharges in patients reporting to clinics and health centres in this rural area of southwestern Uganda. The cure rate of syndromic treatment was remarkably high in this study, supporting the case for syndromic management, which has been a major step forward in rationalising and improving the management of STIs. This information will be useful nationally for refining treatment algorithms. In addition, the low proportion of genital ulcers associated with syphilis or chancroid, and the high proportion of genital ulcers associated with HSV2 infection, which was not covered by our treatment regimen, may help to explain why the STI intervention was ineffective in reducing HIV incidence in the Masaka Intervention Trial. ${ }^{12}$

\section{ACKNOWLEDGEMENTS}

This study was funded by the Medical Research Council (UK) and was conducted through the Medical Research Council Programme on AIDS at the Uganda Virus Research Institute. The authors would like to acknowledge and appreciate the input and assistance offered to the project by staff at the field station laboratory, staff at the general clinic, Kalungu and Bukulula Health Centres, and the participants in the study. We are grateful to Ian Maclean (University of Manitoba, Canada) for the provision of reagents and material for the multiplex polymerase chain reaction tests.

\section{CONTRIBUTORS}

Study concept and design: JW, DM, PM; data collection and interpretation: $\mathrm{PH}, \mathrm{MK}, \mathrm{DM}, \mathrm{BM}, \mathrm{LVdP}, \mathrm{JP}$; statistical analysis: JP; paper drafting: JP, JW; manuscript comment and approval: all authors. 


\section{Authors' affiliations}

J M Pickering, J A G Whitworth, P Hughes, M Kasse, D Morgan, B Mayanja, L V der Paal, Medical Research Council Programme on AIDS, Uganda Virus Research Institute, PO Box 49, Entebbe, Uganda J A G Whitworth, P Mayaud, London School of Hygiene and Tropical Medicine, Keppel Street, London WCIE 7HT, UK

J M Pickering, Medical Research Council Clinical Trials Unit, 222 Euston Road, London NWI 2DA, UK

Competing interest statement: There are no competing interests related to the research conducted that led to this paper being written, or in the writing of the paper itself.

\section{REFERENCES}

1 World Health Organization. Guidelines for the management of sexually transmitted infections. Geneva: WHO, 2003

2 Fleming D, Wasserheit J. From epidemiological synergy to public health policy and practice: the contribution of other sexually transmitted diseases to sexua transmission of HIV infection. Sex Transm Infect 1999;75:3-17.

3 Grosskurth H, Mosha F, Todd J, et al. Impact of improved treatment of sexually transmitted diseases on HIV infection in rural Tanzania: randomised controlled trial. Lancet 1995:346:530-6.

4 O'Farrell N. Increasing prevalence of genital herpes in developing countries: implications for heterosexual HIV transmission and STI control programmes. Sex Transm Infect 1999;75:377-84

5 Bogaerts J, Ricart CA, Van Dyck E, et al. The etiology of genital ulceration in Rwanda. Sex Transm Dis 1989:16:123-6.

6 Lai W, Chen CY, Morse SA, et al. Increasing relative prevalence of HSV-2 infection among men with genital ulcers from a mining community in South Africa. Sex Transm Infect 2003;79:202-7.

7 WHO. Herpes simplex virus type 2. Programmatic and research priorities in developing countries, Report of a WHO/UNAIDS/LSHTM Workshop, London, 14-16 February 2001. Geneva: WHO/HIV_AIDS/2001, 5, 2001.

8 Moodley P, Sturm PD, Vanmali T, et al. Association between HIV-1 infection the efiology of genital ulcer disease, and response to syndromic management. Sex Transm Dis 2003;30:241-5

9 Paz-Bailey G, Rahman M, Ryan C. Report of an evaluation of the syndromic management algorithms in Botswana, CDC, 2003.

10 Htun Y, Morse SA, Dangor Y, et al. Comparison of clinically directed, disease specific and syndromic protocols for the management of genital ulcer disease in Lesotho. Sex Transm Infect 1998;74(Suppl 1):S23-8.

11 Malonza IM, Tyndall MW, Ndinya-Achola JO, et al. A randomized, doubleblind, placebo-controlled trial of single-dose ciprofloxacin versus erythromycin for the treatment of chancroid in Nairobi, Kenya. J Infect Dis 1999;180:1886-93.

12 Kamali A, Quigley M, Nakiyingi J, et al. A community randomised trial of sexual behaviour and syndromic STI management interventions on HIV-1 transmission in rural Uganda. Lancet 2003;361:645-52.
13 Nugent RP, Krohn MA, Hillier SL. Reliability of diagnosing bacterial vaginosis is improved by a standardised method of gram stain interpretation. J Clin Microbiol 1991;29:297-301.

14 Mayaud P, ka-Gina G, Cornelissen J, et al. Validation of WHO algorithm with risk assessment for the clinical management of vaginal discharge in Mwanza, Tanzania. Sex Transm Infect 1998;74(Suppl 1):S77-84

15 Wawer M, Sewankambo N, Serwadda D, et al. Control of sexually transmitted diseases for AIDS prevention in Uganda: a randomised community trial. Lancet 1999;353:525-35.

16 Wolday D, G-Mariam Z, Mohammed Z, et al. Risk factors associated with failure of syndromic treatment of sexually transmitted diseases among women seeking primary care in Addis Ababa. Sex Transm Infect 2004:80:392-4.

17 Kamya M, Nsubuga P, Grant R, et al. The high prevalence of genital herpes among patients with genital ulcer disease in Uganda. Sex Transm Dis 1995;22:351-4.

18 Grosskurth H, Gray R, Hayes R, et al. Control of sexually transmitted diseases for HIV-1 prevention: understanding the implications of the Mwanza and Rakai trials. Lancet 2000;355:1981-7.

19 Korenromp EL, Bakker L, De Vlas SJ, et al. Sexual behaviour changes and genital herpes in Africa. Sex Transm Dis 2002;29:228-38.

20 Wald A, Corey L. How does herpes simplex virus type 2 influence human immunodeficiency virus infection and pathogenesis? J Infect Dis 2003:187:1509-12.

21 Pujades Rodriguez M, Obasi A, Mosha F, et al. Herpes simplex virus type 2 infection increases HIV incidence: a prospective study in rural Tanzania. AIDS 2002;16:451-62.

22 WHO and UNAIDS. Herpes simplex virus type 2: programmatic and research priorities in developing countries. Report of a WHO/UNAIDS/LSHTM workshop, London, 14-16 February 2001.WHO/HIV_AIDS/2001.05

23 Corey L, Wald A, Pate R, et al. Once-daily valacyclovir to reduce the risk of transmission of genital herpes. N Engl J Med 2004;350:11-20.

$24 O^{\prime}$ Farrell N. Soap and water prophylaxis for limiting genital ulcer disease and HIV-1 infection in men in sub-Saharan Africa. Genitourin Med 1993;69:297-300.

25 Morgan D, Mahe C, Mayanja B, et al. Genital ulceration inrural Uganda: sexual activity, treatment-seeking behaviour and the implications for HIV control. Sex Transm Dis 2001;28:431-6.

26 Safrin S. Treatment of acyclovir-resistant herpes simplex virus infections in patients with AIDS. J Acquir Immune Defic Syndr 1992;5(Suppl 1): S29-32.

27 Moodley P, Wilkinson D, Connolly C, et al. Influence of HIV-1 coinfection on effective management of abnormal vaginal discharge. Sex Transm Dis 2003:30: $1-5$

28 Fonck K, Kidula N, Jaoko W, et al. Validity of the vaginal discharge algorithm among pregnant and non-pregnant women in Nairobi, Kenya. Sex Transm Infect 2000;76:33-8.

29 Feldblum $\mathbf{P}$, Kuyoh $M$, Omari $M$, et al. Baseline STD prevalence in a community intervention trial of the female condom in Kenya. Sex Transm Infect 2000;76:454-6. 